Daniela A. S. Bôlla

Programa de Pós-Graduação em Ecologia do Instituto Nacional de Pesquisas da Amazônia. E-mail: danielabolla@hotmail.com

Karoline Ceron

Programa de Pós-Graduação em Ecologia da Universidade Federal de Mato Grosso do Sul. E-mail: adenomera@gmail.com

\section{Fernando Carvalho}

Programa de Pós-Graduação em Ciências Ambientais da

Universidade do Extremo Sul Catarinense. E-mail:

f.carvalho@unesc.net

Denise Lidorio de Mattia

Programa de Pós-Graduação em Ciências Ambientais da

Universidade do Extremo Sul Catarinense. E-mail: de_mattia@hotmail.com

Micheli Ribeiro Luiz Projeto Felinos do Aguaí. Email: micheli@rotasdosul.com

\section{Keite Arcaro Panatta}

Pós-Graduação em Manejo de Recursos Naturais da

Universidade do Extremo Sul Catarinense. E-mail:

keitepanatta@hotmail.com

Diego Dias Pavei

Programa de Pós-Graduação em Arqueologia da Universidade Federal de Pelotas. E-mail: diego.pavei@unesc.net

\section{Rodrigo Ávila Mendonça} Universidade do Sul de Santa Catarina. E-mail:

mendonca.rodrigo@unisul.br

Jairo José Zocche

Programa de Pós-Graduação em Ciências Ambientais da

Universidade do Extremo Sul Catarinense. E-mail: jjz@unesc.net

\section{MASTOFAUNA TERRESTRE DO SUL DE SANTA CATARINA: MAMÍFEROS DE MÉDIO E GRANDE PORTE E VOADORES}

\section{TERRESTRIAL MAMMALS OF SOUTHERN SANTA CATARINA: MEDIUM, LARGE MAMMALS AND BATS}

\section{RESUMO}

Para os estados que compõem a região do Brasil, Santa Catarina é aquele que detém o menor conhecimento sobre a composição de sua mastofauna terrestre, característica esta que é ainda mais acentuada na região sul catarinense, onde poucos estudos foram desenvolvidos. O presente trabalho fornece uma lista atualizada de mamíferos terrestres de médio, grande porte e de morcegos, com ocorrência confirmada por município componente da região sul do estado de Santa Catarina, Brasil. Para o registro das taxas foram utilizados os protocolos de: amostragem em campo; registro bibliográfico da ocorrência das taxas na área de estudo teve por base artigos, dissertações e monografias, que foram obtidos em base de dados online. Um total de 10 ordens e 62 espécies foi registrado para a região, sendo Chiroptera a mais rica ( $\mathrm{n}=29 \mathrm{spp}$.), seguida por Carnívora ( $\mathrm{n}=12 \mathrm{spp}$.), Artiodactyla e Rodentia ( $\mathrm{n}$ = 5 spp. cada). Dentre as espécies registradas, 14 estão enquadradas em pelo menos uma das categorias de ameaça. A região sul catarinense possui importância para a conservação da mastofauna abrigando espécies tanto florestais quanto de ambientes abertos, o que a caracteriza como ecótono entre os Campos de Altitude e a Planície Costeira, ao mesmo tempo em que funciona como corredor de dispersão para a mastofauna sulina.

Palavras-chave: Biodiversidade; mamíferos; morcegos; região sul; riqueza.

\section{ABSTRACT}

For the states that compose the Brazil region, Santa Catarina is the one with the smallest terrestrial Mammals knowledge, being this characteristic even more accentuated in southern of this state, where few studies had been carried out. The present study gives an updated list of medium and large-sized terrestrial Mammals and of bats with confirmed occurrence by municipality of South region to Santa Catarina state, Brazil. To record the Taxa, sampling protocols were used in the field. The bibliographic record of occurrence of the taxa was based on articles, dissertations and monographs got on online database. A total of 10 orders and 62 species was recorded for the region, being Chiroptera the richest $(n=29 \mathrm{spp}$.), followed by Carnivora $(n=12$ spp.), Artiodactyla e Rodentia ( $n=5 \mathrm{spp}$. each). Among the species recorded, 14 are classified at least in one of the threats categories. The Southern region of Santa Catarina has great importance for the conservation of the Mammals sheltering both forest and open-environment species, which characterizes as Ecotone between the Altitude Fields (Campos de Altitude) and Coastal Plain (Planície Costeira), at the same time that it works as dispersion corridor for Mammals of Southern region.

Keywords: Biodiversity, mammals, bats, southern region, richness 


\section{INTRODUÇÃO}

A Mata Atlântica é considerada um dos biomas mais diversos e com alto nível de endemismo. Ocorria originalmente da porção norte do estado do Rio Grande do Norte até a porção sul do Rio Grande do Sul (IBGE, 2012), todavia, atualmente restam menos de 11,73\% da sua cobertura original (Ribeiro et al., 2009). Dentre suas fitofisionomias, a Floresta Ombrófila Densa apresenta maior diversidade em Santa Catarina, em decorrência das variações atitudinais e latitudinais (Klein, 1978; IBGE, 2012). Embora seja uma área de interesse conservacionista, a maior parte de seus remanescentes é composta por espécies pioneiras e secundárias, sendo a fragmentação sua principal ameaça (Schorn et al., 2012; Vibrans et al., 2013). No sul do Estado, áreas originalmente cobertas por Floresta Ombrófila Densa são intensamente ocupadas por atividades antrópicas (Sevegnani et al., 2013), o que resulta no quadro atual de fragmentação.

A região sul de Santa Catarina sofre pressões antrópicas desde o século XVIII, a partir da chegada dos primeiros imigrantes europeus (Piazza e Hübener, 1983), os quais iniciaram a instalação das primeiras vilas e a produção agrícola. Já na metade do século XX, iniciou-se a produção de carvão mineral, a qual alavancou a economia e degradou extensão de 2.000 a 6.000 ha (Alexandre, 1999). No início do século XXI a densidade demográfica desta área superou a média estadual, atingindo 95 habitantes $/ \mathrm{km}^{2}$ (Santa Catarina, 2013). Atualmente, a região se destaca no cenário estadual quanto ao cultivo de arroz irrigado, o qual responde por $59 \%$ da produção catarinense (EPAGRI/CEPA, 2007).

Entre os três estados da região sul do Brasil, Santa Catarina é aquele que historicamente, detém o menor conhecimento sobre a composição de sua mastofauna (Ávilla-Pires 1999). Dentre os trabalhos mais abrangentes sobre a mastofauna catarinense podemos destacar: Marinho-Filho (1996), que apresenta a lista de espécies de morcegos ocorrentes no Estado; Cimardi e Brettas (1996) apresentam a lista da mastofauna registrada e; por fim, o de Cherem et al., (2004) que trazem "a lista dos mamíferos do estado de Santa Catarina, no sul do Brasil", na qual são registradas 152 espécies nativas com registros confirmados e 60 como de provável ocorrência no território catarinense.

Nos anos subsequentes, o número de publicações vem aumentando, especialmente na porção norte do Estado (p. ex.: Althoff, 2007; Cherem et al., 2007; Cherem et al., 2011; Kuhnen et al., 2011; Oliveira-Santos et al., 2012; Tortato et al., 2014; Orlandin et al., 2015). Na região sul de Santa Catarina as lacunas no conhecimento da composição da mastofauna são ainda maiores, haja vista o baixo número estudos realizados (p.ex.: Cherem et al., 2004; Cherem, 
2005). Entretanto, nos últimos anos têm-se observado incremento nas publicações, principalmente para a ordem Chiroptera (p.ex.: Carvalho et al., 2008; 2009; Zocche et al., 2010; Carvalho e Fabián, 2011; Carvalho et al., 2013; 2014; 2017a, b; Bôlla et al., 2017).

Considerando a intensificação das atividades antrópicas, sobretudo a crescente urbanização e seus impactos sobre o bioma Mata Atlântica em Santa Catarina, inventários sobre sua fauna são imprescindíveis, para que se possa compreender melhor os padrões de distribuição das espécies, a fim de se adotar políticas de manejo de proteção (Penter et al., 2008). Inventários sobre a mastofauna em regiões pouco estudadas, podem fornecer subsídios para a conservação e para o manejo de áreas indispensáveis à manutenção das populações silvestres ali existentes. Assim sendo, o presente estudo tem por objetivo fornecer uma lista atualizada de espécies de mamíferos de médio e grande porte e de morcegos com ocorrência documentada na região sul de Santa Catarina, sul do Brasil.

\section{MATERIAL E MÉTODOS}

O estudo foi desenvolvido na região sul do estado de Santa Catarina, a qual abrange 44 municípios (Fig.1). A região está inserida no Bioma Mata Atlântica, nas regiões fitogeográficas da Floresta Ombrófila Densa das Terras Baixas, Submontana e Montana e Formação Pioneira com Influência Marinha (Restinga) (Klein 1978; IBGE, 2012).

Figura 1. Localização do Estado de Santa Catarina na região sul do Brasil e localização dos municípios que compõem a região sul do Estado de Santa Catarina

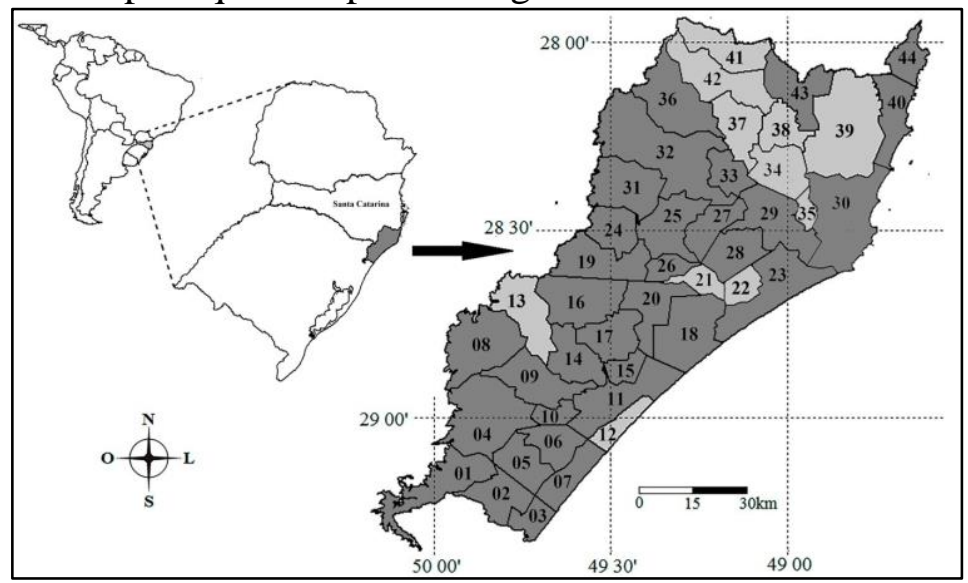

Fonte: autores, 2016

Legenda: a cor cinza claro destaca os municípios onde não houveram registros de ocorrência de mamíferos de médio/grande porte e voadores e, a cor cinza escuro destaca os municípios onde houveram registros.

O clima é do tipo Cf conforme classificação climática de Köppen (Alvares et al., 2013), ocorrendo as duas variações climáticas a e b. A temperatura média anual oscila entre 11,4 a $19,3{ }^{\circ} \mathrm{C}$, as temperaturas médias máximas e mínimas variam, respectivamente de 16,9 a 25,9 
${ }^{\circ} \mathrm{C}$ e de 7,6 a $15,1{ }^{\circ} \mathrm{C}$. A precipitação pluviométrica total anual é de 1.220 a $1.660 \mathrm{~mm}$, com o total anual de 102 e 150 dias (EPAGRI, 2001).

\section{Obtenção dos Dados}

Para o registro da ocorrência dos taxa foram utilizadas diferentes fontes de dados, tais como: amostragens em campo, oriundas de projetos desenvolvidos pelo Laboratório de Ecologia de Paisagem e de Vertebrados da UNESC e pelo Instituto Felinos do Aguaí; material depositado na coleção do Museu de Zoologia Profa ${ }^{a}$ Morgana Cirimbelli Gaidzinski da Universidade do Extremo Sul Catarinense; registros obtidos no SpeciesLink e; revisão bibliográfica.

Para mamíferos de médio e grande porte, a obtenção dos dados em campo envolveu diferentes métodos de amostragem, os quais incluem: armadilhamento fotográfico (câmerastrap); registros indiretos por meio de vestígios (fezes e pegadas) e; busca por animais atropelados em rodovias da região. Também foram considerados os registros oportunísticos, tais como encontro de carcaças de animais atropelados, e aqueles obtidos durante amostragens com enfoque em outros grupos de vertebrados. A identificação dos registros indiretos seguiu Pitman et al. (2002), De Oliveira e Cassaro (2005), Angelo et al. (2008), Carvalho Júnior e Luz (2008) e Becker e Dalponte (2013).

Para morcegos, os dados inéditos resultaram de amostragens para estudos pontuais, os quais foram desenvolvidos pelos autores na região sul de Santa Catarina. Como métodos de amostragem foram utilizadas redes de neblina instaladas ao nível do solo e captura manual em abrigos diurnos. A identificação taxonômica dos quirópteros seguiu Eger (2007), MarquesAguiar (2007) e Miranda et al. (2011).

O registro bibliográfico da ocorrência dos taxa na área de estudo teve por base artigos, dissertações e monografias, obtidos em base de dados online (SpeciesLink: http://splink.cria.org.br), assim como nos acervos digitais das bibliotecas da Universidade do Extremo Sul Catarinense, Universidade Federal do Rio Grande do Sul, Universidade Federal de Santa Catarina e Universidade Regional de Blumenau. A busca nos bancos de dados foi realizada utilizando as seguintes palavras-chaves: mamíferos; mastofauna, Santa Catarina, extremo sul Catarinense; inventário; riqueza; Artiodactyla, Carnivora, Chiroptera, Cingulata, Lagomorpha, Pilosa, Primates e Rodentia. Essas palavras foram utilizadas de forma individual ou em combinação, sendo estas repetidas em todos os bancos de dados online.

Posteriormente, foi realizada triagem para todas as referências, sendo desconsiderados registros de espécies sem distribuição confirmada para Santa Catarina, assim como, taxa com 
identificação não conclusiva. Foi considerado como identificação não conclusiva, aquele táxon que a identificação ao nível de espécie depende de análise de estruturas morfológicas internas (crânio), sendo este procedimento, não descrito nos métodos do estudo. A nomenclatura taxonômica dos diferentes grupos seguiu Paglia et al., (2012). Para analisar o status de conservação foi utilizada a lista vermelha da IUCN (IUCN, 2015) em âmbito global, a Lista da Fauna Brasileira Ameaçada de Extinção (MMA, 2014) em âmbito nacional e a Lista de Espécies Ameaçadas de Extinção de Santa Catarina em âmbito estadual (CONSEMA, 2011).

\section{RESULTADOS}

Dentre os 44 municípios que compõem a Região Sul de Santa Catarina, em 33 deles obteve-se ao menos um registro de ocorrência de mamífero terrestre (Fig. 1). Em conjunto, dados inéditos e bibliográficos somam 10 ordens e 62 espécies com ocorrência confirmada na área de estudo (Tabela 1). Chiroptera foi a ordem com maior riqueza ( $\mathrm{n}=29 \mathrm{spp}$.), seguida por Carnivora ( $n=12$ spp.), Artiodactyla e Rodentia ( $n=5$ spp. cada).

Figura 2: Espécies registradas na região sul de Santa Catarina, enquadradas em uma das categorias de ameaça em âmbito global, nacional e/ou estadual.
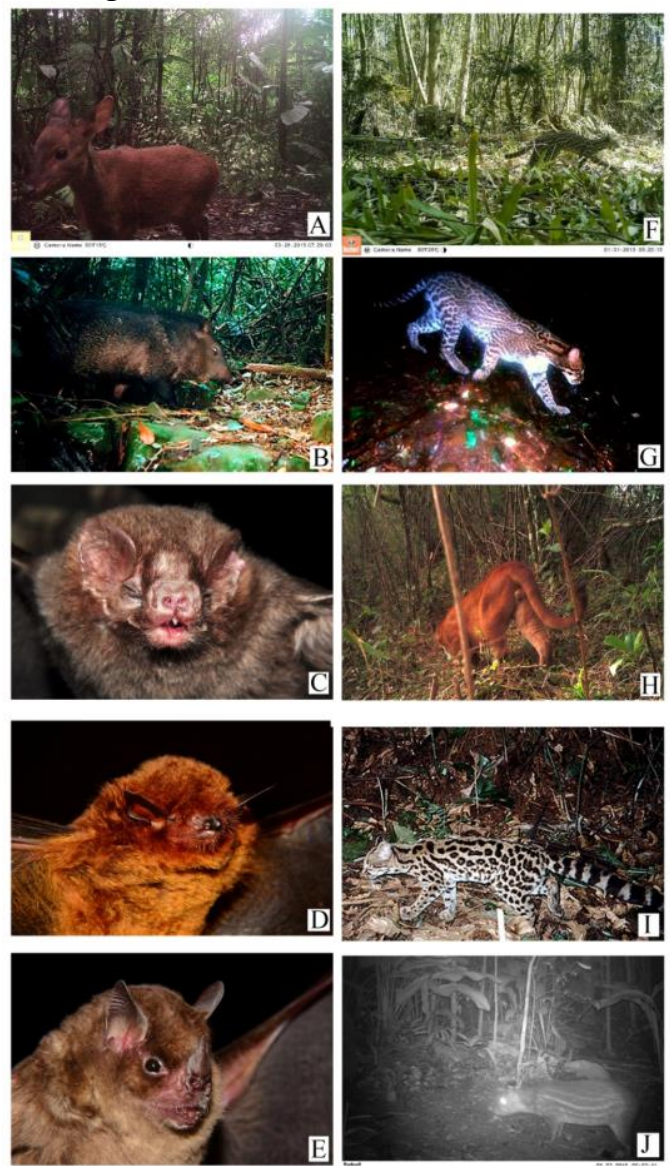

Legenda: A - Mazama nana; B - Pecari tajacu; C - Diphylla ecaudata; D - Myotis ruber; E - Sturnira tildae; F - Leopardus guttulus; G - Leopardus pardalis; H - Puma concolor; I - Leopardus wiedii; J - Cuniculus paca Fonte: autores, 2016 
Os municípios que comportaram maior riqueza foram: Pedras Grandes ( $\mathrm{n}=40 \mathrm{spp}$.), Urussanga ( $\mathrm{n}=32$ spp.), Siderópolis ( $\mathrm{n}=31$ spp.), Timbé do Sul, Nova Veneza e Treviso $(\mathrm{n}=$ 30 spp. cada) (Tabela 1). Cerdocyon thous $(\mathrm{n}=22)$, Hydrochoerus hydrochaeris $(\mathrm{n}=20)$, Procyon cancrivorus $(\mathrm{n}=19)$ e Didelphis albiventris $(\mathrm{n}=19)$ foram as espécies registradas em maior número de municípios. Quatorze espécies, dentre as 61 registradas, se encontram sob uma categoria de ameaça, sendo, cinco em âmbito global, oito em âmbito nacional e 11 em nível estadual (Figura 2; Tabela 2).

\section{DISCUSSÃO}

Apesar dos diversos impactos ambientais presentes na região sul de Santa Catarina, sua mastofauna terrestre mostra-se ainda diversificada. O número de espécies observado no presente estudo corresponde a 69\% das espécies de mamíferos terrestres do Estado (Cherem et al., 2004; Miranda et al., 2006; Miranda et al., 2007; Passos et al., 2010; Carvalho e Fabián, 2011). A esta alta representatividade atribui-se dois fatores principais: o primeiro está relacionado a abrangência da área de estudo, que engloba diversas fitofisionomias da Mata Atlântica e ecossistemas associados e; o segundo resulta do longo período e das formas diversificadas de obtenção dos dados, uma vez que os registros se deram em vários estudos desenvolvidos por diferentes pesquisadores em momentos distintos. Ambientes complexos, como os presentes na área estudada, e que abrigam diversos micro-habitats tendem a comportar maior riqueza, quando comparados com ambientes homogêneos (Silva et al., 2011). Além disso, trabalhos de longo prazo, mesmo com amostragens não sistematizadas tendem a registrar grande riqueza (p.ex.: Cherem et al., 2011, Câmara e Oliveira 2012, Tortato et al., 2014).

Em 75\% dos municípios da Região Sul de Santa Catarina houve ao menos um registro de mamíferos de médio, grande porte e/ou morcegos. Dentre os 11 municípios sem nenhum registro, sete estão localizados na porção norte da Região Sul de Santa Catarina e ao sul do Parque Estadual da Serra do Tabuleiro. Essa unidade de conservação é a maior de Santa Catarina (Cherem et al., 2011), sendo o limite austral de distribuição de muitas espécies tropicais (FATMA, 2000). Essa lacuna de registros na porção norte da área do presente estudo deve-se principalmente, ao menor esforço amostral empregado nessa região, o que a torna prioritária para a realização de trabalhos que visem a obtenção de dados primários acerca de sua mastofauna.

Em contrapartida, os municípios da porção central e dos encraves da Serra Geral (p. ex. Pedras Grandes, Urussanga, Siderópolis e Timbé do Sul) apresentaram elevada riqueza. Isto se 
justifica pelo maior esforço amostral despendido nestes municípios, uma vez que abrigam áreas de proteção ambiental (p.ex. Pedras Grandes - Parque Ecológico e Ecoturístico de Pedras Grandes, Siderópolis - REBIO Aguaí e Barragem do Rio São Bento, Orleans/Grão-Pará Parque Estadual da Serra Furada). Nessas áreas, foram realizados inventários da fauna para o desenvolvimento de projetos de pesquisa, monografias, dissertações e instalação de grandes empreendimentos (p.ex. Siderópolis - Barragem do Rio São Bento e Timbé do Sul - Barragem do Rio do Salto). Ainda, a proximidade de alguns municípios com as instituições de ensino e de pesquisa facilita a realização de amostragens de campo, consequentemente, aumento a riqueza registrada nestes municípios.

Sendo a ordem mais diversa, Chiroptera comportou $46 \%$ do total de espécies registradas no presente estudo. Esta dominância é um padrão comum em muitas assembleias de mamíferos em diferentes ambientes no Brasil (p.ex.: Alho et al., 2011; Cherem et al., 2011; MarquesAguiar et al., 2002). Morcegos correspondem à segunda ordem com maior riqueza dentre os mamíferos (Kalko, 1998), perdendo somente para Rodentia (Wilson e Reeder 2005). No Brasil, quirópteros correspondem a aproximadamente $25 \%$ da mastofauna terrestre registrada (Reis et al., 2011), já para Santa Catarina este percentual é de 30\% (Cherem et al., 2004). Todavia, em algumas localidades este grupo pode contribuir com até 50\% da composição da mastofauna, influenciando fortemente os padrões de riqueza e diversidade (Estrada e Coates-Estrada, 2002). A pequena representatividade de Rodentia na amostra, deve-se ao fato desta ordem ser composta principalmente, por espécies de pequeno porte (Oliveira e Bonvicino,2011), as quais não foram incluídas no presente estudo.

A alta frequência de Cerdocyon thous, Hydrochoerus hydrochaeris, Procyon cancrivorus e Didelphis albiventris sugere que estas espécies são amplamente distribuídas e abundantes na Região Sul de Santa Catarina, assim como observado em outras áreas da Mata Atlântica (p.ex.: Cherem et al., 2007, Cáceres et al., 2008, Onghero Jr. et al., 2012, Tortato et al., 2012). Todas essas espécies são classificadas como generalistas, as quais se adaptam facilmente aos ambientes com diferentes níveis de antropização (p.ex.: Cabrera e Yepes 1960, Cáceres 2002, Canevari e Vaccaro, 2007).

De forma contrária, 11 espécies foram registradas em apenas um município, sendo seis pertencentes à Chiroptera. Para esse grupo, a baixa frequência de Diphylla ecaudata, Mimon bennettii e Platyrrhinus recifinus pode estar relacionada ao fato de que o sul de Santa Catarina é o limite austral de distribuição das mesmas (Kwon e Gardner 2007; Carvalho et al., 2008; Carvalho e Fabián 2011; Carvalho et al., 2013), o que as torna naturalmente raras nessa região. Já Myotis dinelli e Chrotopterus auritus são espécies pouco frequentes em inventários de 
morcegos na região sul do Brasil (p.ex.: Bianconi et al., 2004; Althoff 2007; Carvalho et al., 2013), assim como observado no presente estudo. Já o registro de Lasiurus cinereus no município de Tubarão, corresponde ao segundo registro da espécie para o Estado, que até então era conhecida apenas para a localidade de Água Doce, no oeste de Santa Catarina (Cherem; Althoff 2015). O registro em Tubarão é relevante também pelo fato de representar uma ampliação da área de distribuição conhecida para espécie (Gardner; Handley 2007).

O baixo número de registros de Ozotoceros bezoarticus, Lycalopex gymnocercus e Conepatus chinga deve-se, principalmente, à distribuição predominante em áreas abertas e de altitude (Canevari e Vaccaro, 2007; Cherem et al., 2007; Miranda et al., 2008; Tortato e Althoff, 2011). Essas áreas são pouco representativas no sul do Estado e foram pouco contempladas neste estudo. Sylvilagus brasiliensis foi citada apenas através de registro histórico (Cherem et al., 2004), não havendo nenhum registro atual da espécie na região. Apesar de possuir hábitos solitários e ser de difícil visualização (Canevari e Vaccaro, 2007), não é descartada a possibilidade de a espécie estar extinta regionalmente. Fatores que levariam a extinção da mesma incluem, a redução e perda de seu habitat, a introdução de espécies exóticas competidoras (Lepus europaeus) e a caça, que mesmo proibida em todo território nacional (Brasil 1998), ainda é praticada no Estado e particularmente na região estudada, com alta intensidade.

Seis espécies que foram registradas em apenas um município, constam nas listas de espécies ameaçadas, seja em nível global, nacional ou estadual. Para os mamíferos de Artiodactyla a caça e a fragmentação ainda figuram como principais ameaças à manutenção de suas populações (Costa et al., 2005; Di Bitetti et al., 2008; Braga e Kuniyoshi, 2010). Já para as espécies de Felidae, Chiroptera, Primata e Rodentia, a alteração ambiental e a fragmentação de florestas figuram como as maiores ameaças (Costa et al., 2005). Para algumas espécies de mamíferos de grande porte, como Leopardus pardalis, Puma concolor e Puma yagouarondi, que necessitam de grandes áreas para sobreviver, a fragmentação de seus habitats reduziu o tamanho de suas populações, visto que pequenos fragmentos são insuficientes em recursos para a sobrevivência de suas populações em longo prazo (Chiarello, 1999; Costa et al., 2005).

A região sul catarinense possui grande importância para a conservação da mastofauna, tanto para o estado de Santa Catarina quanto para o Rio Grande do Sul, visto sua localização limítrofe entre os estados, assim como, o estado de conservação de suas florestas localizadas nas encostas da Serra Geral. A ocorrência de oito espécies ameaçadas em nível nacional, 11 em nível estadual e a presença de espécies tanto florestais quanto de áreas abertas demonstram a 
importância da região como área de ecótono entre diferentes ambientes da Mata Atlântica, servindo de abrigo e corredor de dispersão para a mastofauna sulina.

\section{AGRADECIMENTOS}

Os autores agradecem à Vanessa Matias Bernardo, Fábio Hammen Lhanos, João Paulo Gava-Just, Rafael Romagna pelos dados cedidos sobre a ocorrência da mastofauna na região Sul; Ao Museu de Zoologia Profa. Morgana Cirimbelli Gaidzinski pelo acesso a coleção; à Beatriz Lima Luciano e a Gabriel Preuss Custódio pelo auxílio com a formatação do texto e referências, à CAPES pelas bolsas de estudo concedidas à DLM e à KC e ao CNPq pela bolsa de Iniciação Científica à DASB.

\section{REFERENCIAS}

ALEXANDRE, N. Z. Diagnóstico ambiental da região carbonífera de Santa Catarina: degradação dos recursos naturais. Revista Tecnolia e Ambiente, v. 5, p.35-53, 1999.

ALHO, C. J. R.; CAMARGO, G.; FISCHER, E. Terrestrial and aquatic mammals of the Pantanal. Brazilian. Journal of Biology, v.71, n.1, p.297-310, 2011.

ALVARES, C. A.; STAPE, J. L.; SENTELHAS, P. C.; MORAES, G.; LEONARDO, J.; SPAROVEK, G. Köppen's climate classification map for Brazil. Meteorologische Zeitschrift, v.22, n. 6, p.711-728, 2013.

ANGELO, C. D.; PAVIOLO, A.; BITETTI, M. D.; CHIAPPE, A. Guías de Huellas: De Los Mamíferos de Misiones Y Otras Áreas del Subtrópicos de Argentina. Argentina: Subtrópico, 2008. 112p.

ÁVILA-PIRES, F. D. Mamíferos descritos do Estado de Santa Catarina. Revista Brasileira de Zoologia, v.16, n.2, p.51-62, 1999.

BARROS-FERRAZ, K. M. P. M.; BARROS-FERRAZ, S. F.; MOREIRA, J. R.; COUTO, H. T. Z.; VERDADE, L. M. Capybara (Hydrochoerus hydrochaeris) distribution in agroecosystems: a cross-scale habitat analysis. Journal of Biogeography, v.34, n.2, p.223-230, 2007.

BECKER, M.; DALPONTE, J. C. Rastros de Mamíferos Silvestres Brasileiros - Um Guia de Campo. Brasília: Technical Books, 2013. 166p.

BELOLLI, M.; QUADROS, J.; GUIDI, A. História do carvão de Santa Catarina: 1790-1950. Imprensa Oficial do Estado de Santa Catarina, Criciúma, 2002. 297p.

BOLLA, D. A. S.; CARVALHO, F.; MIRANDA, J. M. D.; ZOCCHE, J. J.; HARTERMARQUES, B.; MARTINS, R.; PAVEI, D. D.; LUZZIETTI, M. M. Assembleia de morcegos (Mammalia: Chiroptera) em ambiente de Restinga alterada no sul do Brasil. Neotropical Biology and Conservation, v.12, n.2, p.135-142, 2017.

BRAGA, F. G.; KUNIYOSHI, Y. S. Estimativas de parâmetros populacionais e demográficos de Ozotoceros bezoarticus (Artiodactyla, Cervidae) em Piraí do Sul, Paraná, sul do Brasil. Iheringia, v.100, p.105-110, 2010. 
BRASIL. Lei 9.605 de 12 de fevereiro de 1998. Dispõe sobre as sanções penais e administrativas derivadas de condutas e atividades lesivas ao meio ambiente e dá outras providências, 1998.

BRITO, D.; OLIVEIRA, L. C.; OPREA, M.; MELLO, M. A. R. An overview of Brazilian mammalogy: trends, biases and future directions. Zoologia, v.26, p.67-73, 2009.

CABRERA, A.; YEPES, J. Mamíferos Sudamericanos. Vida, costumes y descripción. Buenos Aires: Ediar. 1960. 370p.

CÁCERES, N. C. Food Habits and Seed Dispersal by the White-Eared Opossum, Didelphis albiventris, in Southern Brazil. Studies on Neotropical Fauna and Environment, v.37, n.2, p.97-104, 2002.

CÁCERES, N.; BORNSCHEIN, M.; LOPES, W. Uso do hábitat e a conservação de mamíferos no sul do bioma Cerrado. In: REIS, N. R.; PERACCHI, A. L.; SANTOS, G. A. S. D. (Eds.). Ecologia de mamíferos. Londrina. 2008. p.123-132.

CÂMARA, E. M. V. C.; OllVEIRA, L. C. Mammals of Serra do Cipó National Park, southeastern Brazil. Check List, v.8, n.3, p.355-359, 2012.

CANEVARI, M.; VACCARO, O. Guía de mamíferos del sur de América del Sur. Buenos Aires: Lola, 2007. 413p.

CARVALHO, F.; BOLLA, D. A. S.; MIRANDA, J. M. D.; ZOCCHE, J. J. Deslocamentos de morcegos frugívoros (Chiroptera: Phyllostomidae), entre diferentes fitofisionomias da Mata Atlântica, no Sul do Brasil. Revista Brasileira de Biociências, v.15, n.2, p. 78-82, 2017a.

CARVALHO, F.; BOLLA, D. A. S.; PATEL, F. M.; MIRANDA, J. M. D.; ALTHOFF, S. L.; ZOCCHE, J. J. Ampliação de distribuição de Eumops patagonicus Thomas, 1924, (Chiroptera: Molossidae) e primeiro registro em ambiente de Restinga na Costa Leste do Brasil. Mastozoología Neotropical, v.24, p.1-8, 2017.

CARVALHO, F.; CRUZ-NETO, A. P.; ZOCCHE, J. J. Ampliação da distribuição e descrição da dieta de Mimon bennettii (Phyllostomidae: Phyllostominae) no sul do Brasil. Chiroptera Neotropical, v.14, n.2, p.403-407, 2008.

CARVAlHO, F.; FABIÁN, M. E. Mammalia, Chiroptera, Phyllostomidae, Platyrrhinus recifinus (O. Thomas, 1901): first record in the state of Santa Catarina, Southern Brazil. CheckList, v.7, n.2, p.139-141, 2011.

CARVALHO, F.; FABIÁN, M. E; MENEGHETI, J. O. Vertical structure of an assemblage of bats (Mammalia: Chiroptera) in a fragment of Atlantic Forest in Southern Brazil. Zoologia, v. 30, n.5, p.491-498, 2013.

CARVALHO, F.; FABIAN, M. E.; MENEGHETI, J. O. Variação sazonal no número de capturas de Artibeus lituratus (Olfers, 1818) e Sturnira lilium (E. Geoffroy, 1810) (Chiroptera: Phyllostomidae) no estrato superior de um remanescente de Mata Atlântica no sul do Brasil.

Biotemas, v. 27, p.131-138, 2014.

CARVALHO, F.; ZOCCHE, J. J.; MENDONÇA, R. A. Morcegos (Mammalia, Chiroptera) em restinga no município de Jaguaruna, sul de Santa Catarina, Brasil. Biotemas, v.22, n.3,193-201, 2009.

CARVALHO-JÚNIOR, O.; LUZ, N. Pegadas: série boas práticas. Belém: Editora da Universidade Federal do Pará, 2008. 64p. 
CHEREM, J. J. Registros de mamíferos não voadores em estudos de avaliação ambiental no sul do Brasil. Biotemas, v.18, n.2, p.169-202, 2005.

CHEREM, J. J.; ALTHOFF, S. L. Mamíferos de uma área de estepe ombrófila nos estados do Paraná e Santa Catarina, sul do Brasil. Boletim da Sociedade de Mastozoologia, v.72, p.42$50,2015$.

CHEREM, J. J.; GRAIPEL, M. E; TORTATO, M. Mastofauna terrestre do Parque Estadual da Serra do Tabuleiro, Estado de Santa Catarina, sul do Brasil. Biotemas, v.24, n.3, p.73-84, 2011.

CHEREM, J. J.; KAMMERS, M.; MARTINS, A. Mamíferos de médio e grande porte atropelados em rodovias do Estado de Santa Catarina, Sul do Brasil. Biotemas, v.20, p.81-96, 2007.

CHEREM, J. J.; SIMÕES-LOPES, P. C.; ALTHOFF, S.; GRAIPEL, M. E. Lista dos mamíferos do estado de Santa Catarina, sul do Brasil. Mastozoología Neotropical, v.11, n.2, p.151-184, 2004.

CHIARELLO, A. G. Effects of fragmentation of the Atlantic forest on mammal communities in south-eastern Brazil. Biological Conservation, v.89, n.1, p.71-82, 1999.

CIMARDI, A. V; BRETTAS, E. P. Mamíferos de Santa Catarina. Florianópolis, Fatma. 1996. 302p.

CONSEMA. Reconhece a Lista Oficial de Espécies da Fauna Ameaçadas de Extinção no Estado de Santa Catarina e dá outras providências. Resolução Consema $\mathrm{n}^{\circ} 002$, de 06 de dezembro de 2011. Florianópolis: Diário Oficial de Santa Catarina. p.2-8, 2011.

COSTA, L. P.; LEITE, Y. L. R.; MENDES, S. L.; DITCHFIELD, A. D. Mammal conservation in Brazil. Conservation Biology, v.19, n.3, p.672-679, 2005.

DE OLIVEIRA, T. G.; CASSARO, K. Guia de campo dos felinos do Brasil. São Paulo: PróVida Brasil, 2005. 80p.

DI BITETTI, M. S.; PAVIOlO, A.; FERRARI, C. A.; ANGElO, C.; BLANCO, Y. Differential responses to hunting in two sympatric species of brocket deer (Mazamaamericana and M. nana). Biotropica, v.40, n.5, p.636-645, 2008.

EGER, J. L. Family Molossidae. In: GARDNER, A. L. Mammals of South America: Marsupials, Xenarthrans, Shrews, and Bats. Chicago: The University of Chicago Press, 2007. p. 400-439.

EPAGRI. Dados e Informações Biofísicas da Unidade de Planejamento Regional Litoral Sul Catarinense - UPR 8. EPAGRI, Florianópolis, 2001.

EPAGRI/CEPA. Síntese Anual da Agricultura de Santa Catarina 2006-2007. Florianópolis. 282 p. Disponível em: <http://www.epagri.sc.gov.br/?s=CEPA〉, 2007.

ESTRADA, A.; COATES-ESTRADA, R. Bats in continuous forest, forest fragments and in an agricultural mosaic habitat-island at Los Tuxtlas, Biological Conservation, Mexico, v.103, n.2, p.237-245, 2002.

FARIA-CORRÊA, M.; BALBUENO,R. A.; VIEIRA, E. M.; FREITAS, T. R. O. Activity, habitat use, density, and reproductive biology of the crab-eating fox (Cerdocyon thous) and comparison with the pampas fox (Lycalopex gymnocercus) in a Restinga area in the southern Brazilian Atlantic Forest. Mammalian Biology - Zeitschriftfür Säugetierkunde, v.74, n. 3, p.220-229, 2009. 
FATMA. Parque Estadual da Serra do Tabuleiro: Diagnóstico dos meios físico e biótico, produto básico de zoneamento. FATMA, Florianópolis, 2000.

FUNDAÇÃO S.O.S MATA ATLÂNTICA e INPE. Atlas dos remanescentes florestais da Mata Atlântica período 1995-2000. Fundação SOS Mata Atlântica, INPE, São Paulo, 2002.

GALETTI, M.; SAZIMA, I. Impact of feral dogs in an urban Atlantic forest fragment in southeastern Brazil. Natureza e Conservação, v.4, n.1, p.146-151, 2006.

GARDNER, A. L.; HANDLEY, C. O. Genus Lasiurus. In: GARDNER, A. L (Ed.) Mammals of South America: marsupials, Xenarthrans, Shrews, and bats. Chicago and London, The University of Chicago Press. 2007. 457-468p.

IBGE. Manual Técnico da Vegetação Brasileira. IBGE, Rio de Janeiro, 2012.

IUCN. The IUCN Red List of Threatened Species, 2015. Disponível em:<http://www.iucnredlist.org/.>Acesso em: 12 de dezembro de 2016.

KALKO, E. Organization and diversity of tropical bat communities through space and time. Zoology, v. 101, n. 4, p.281-297, 1998.

KLEIN, R. M. Mapa Fitogeográfico de Santa Catarina. In: REINZ, R. Flora Ilustrada Catarinense. Herbário Barbosa Rodrigues, Itajaí, 1978. p.24.

KWON, M.; GARDNER, A. L. Subfamily Desmodontinae, In: Gardner, A. L. (Ed). Mammals of South America. Chicago: University of Chicago Press, 2007, p.218-224.

MARQUES-AGUIAR, S. A. Genus Artibeus Lech, 1821. In: GARDNER, A. L. Mammals of South America: Marsupials, Xenarthrans, Shrews, and Bats. Chicago: The University of Chicago Press, 2007. p. 301-321.

MARQUES-AGUIAR, S. A.; MElO, C. C. S.; AGUIAR, G. F. S.; QUEIRÓZ, J. A. L. Levantamento preliminar da mastofauna da região de Anajás-Muaná, Ilha de Marajó, Pará, Brasil. Revista Brasileira de Zoologia. v.19, p.841-854, 2002.

MATTIA, D. L. Atropelamentos de vertebrados silvestres em rodovias do extremo sul e do planalto sul catarinense. 2016. 111f. Dissertação (mestrado em Ciências Ambientais) Universidade do Extremo Sul Catarinense, Criciúma. 2016.

MENEZES, C. D.; WATERKEMPER, K. Evolução dos processos de degradação ambiental resultante da mineração de carvão em Santa Catarina de 1930-1973. In: MILLIOLI, G.; SANTOS, R.; CITADINI-ZANETTE, V. Mineração de carvão, meio ambiente e desenvolvimento sustentável no sul de Santa Catarina: uma abordagem interdisciplinar. Curitiba: Juruá, 2009. p. 205-225.

MIRANDA, J. M. D.; AZEVEDO-BARROS, M. F. M.; PASSOS, F. C. First record of Histiotus laephotis Thomas (Chiroptera, Vespertilionidae) from Brazil. Revista Brasileira de Zoologia, v.24, p.1188-1191, 2007.

MIRANDA, J. M. D.; BERNARDI, I. P.; PASSOS, F. C. Chave ilustrada para a determinação dos morcegos da Região Sul do Brasil. Curitiba, 2011, 51p.

MIRANDA, J. M. D.; PULCHÉRIO-LEITE, A.; MORO-RIOS, R. F.; PASSOS F. C. Primeiro registro de Histiotus montanus (Philippi \& Landbeck) para o Estado do Paraná, Brasil (Chiroptera, Vespertilionidae). Revista Brasileira de Zoologia. v.23, p.584-587, 2006.

MIRANDA, J. M. D.; RIOS, R. F. M.; PASSOS, F. C. Contribuição ao conhecimento dos mamíferos dos Campos de Palmas, Paraná, Brasil. Biotemas, v.21, n.2, p.97-103, 2008. 
MMA. Lista Nacional Oficial de Espécies da Fauna Ameaçadas de Extinção. Portaria n ${ }^{\circ}$ 444, p.121-126, 2014.

OLIVEIRA, J. A.; BONVICINO, C. R. Ordem Rodentia, In: REIS, N. R.; PERACCHI, A. L.; PEDRO, W. A.; LIMA, I. P. (Eds.) Mamíferos do Brasil. $2^{\text {a }}$ Ed. Londrina: Eduel, 2011, p.358406.

ONGHERO, J. R. O.; FAVRETTO, M. A.; PIOVEZAN, J. C.; SPIER, E.; GUZZI, A.; DALAVÉQUIA, M. A. Mamíferos em remanescentes florestais de uma fazenda de plantação de Pinus sp., Água Doce, Santa Catarina, Brasil. Unoesc \& Ciência, v.3, n.1, p.57-64, 2012.

PAGLIA, A. P.; FONSECA, G. A.; RYLANDS, A. B.; HERRMANN, G.; AGUIAR, L. M.; CHIARELlO, A. G.; LEITE, Y. L.; COSTA, L. P.; SICILIANO, S.; KIERULFF, M. C. M. Lista Anotada dos Mamíferos do Brasil, Occasional Papers in Conservation Biology, v, 2: 716, 2012,

PASSOS, F. C.; MIRANDA, J. M. D.; BERNARDI, I. P.; KAKU-OLIVEIRA, N. Y.; MUNSTER, L. C. Morcegos da Região Sul do Brasil: análise comparativa da riqueza de espécies, novos registros e atualizações nomenclaturais (Mammalia, Chiroptera). Iheringia, v. 100, n.1, p. 25-34, 2010.

PENTER, C.; PEDÓ, E.; FABIÁN, M. E.; HARTZ, S. M. Inventário Rápido da Fauna de Mamíferos do Morro Santana, Porto Alegre, RS. Revista Brasileira de Biociências. v.6, n.1, p.117-125, 2008.

PIAZZA, W. F.; HÜBENER, L. M. Santa Catarina: história da gente. Florianópolis: Lunardelli, 1983, 150p.

PITMAN, M. R. P. L.; OLIVEIRA, T. G.; PAULA, R. C.; INDRUSIAK, C. Manual de identificação, prevenção e controle de predação por carnívoros. Edições IBAMA, Brasília, 2002.

REIS, N. R.; PERACCHI, A. L.; PEDRO, W. A.; LIMA, I. P. Mamíferos do Brasil. Londrina: Eduel, 2011, 437p.

RIBEIRO, M. C.; METZGER, J. P.; MARTENSEN, A. C.; PONZONI, P. J.; HIROTA, M. M. The Brazilian Atlantic Forest: How much is left, and how is the remaining forest distributed? Implications for conservation. Biological Conservation, v.142, p.1141-1153, 2009.

SANTA CATARINA. Secretaria de Estado de Assistência Social, Trabalho e Habitação. Boletim regional do Mercado de Trabalho Catarinense. Série 2013, n.05, Mesoregião Sul Catarinense, 2013. Disponível em: <http://www.sst.sc.gov.br/sine/arquivos/BOLETIMREGIONAL.Sul2013.pdf> Acesso em 26 de fevereiro de 2016.

SANTOS, F. M. D.; PELLANDA, M.; TOMAZZONI, A. C.; HASENACK, H.; HARTZ, S. M. Mamíferos carnívoros e sua relação com a diversidade de hábitats no Parque Nacional dos Aparados da Serra, sul do Brasil. Iheringia, v.94, n.3, p.235-245, 2004.

SCHORN, L. A.; GASPER, A. L. D.; MEYER, L.; VIBRANS, A. C. Síntese da estrutura dos remanescentes florestais em Santa Catarina. In: VIBRANS, A.; SEVEGNANI, L.; GASPER, A. L. D.; LINGNER, D. V. D. Inventário Florístico Florestal de Santa Catarina Vol. 1: Diversidade e Conservação dos remanescentes florestais. Blumenau: Edifurb, 2012, p. 125140 .

SEVEGNANI, L.; GASPER, A. L. D.; BONNET, A.; SOBRAL, M. G.; VIBRANS, A. C.; VERDI, M.; SANTOS, A. S. D.; DREVECK, S.; KORTE, A.; SCHMITT, J. Flora Vascular da Floresta Ombrófila Densa em Santa Catarina. In: VIBRANS, A.; SEVEGNANI, L.; GASPER, 
A. L. D.; LINGNER, D. V. D. Inventário Florístico Florestal de Santa Catarina, Vol. IV, Floresta Ombrófila Densa. Blumenau: Edifurb, 2013, p.127-139.

SILVA, R. A.; MARTINS, I. A.; ROSSA-FERES, D. C. Environmental Heterogeneity: Anuran diversity in homogeneous environments. Zoologia. v.5, p.610-618, 2011.

TORTATO, F. R.; ALTHOFF, S. L. Mammalia, Myrmecophagidae, Myrmecophaga tridactyla (Linnaeus, 1758) and Cervidae, Ozotoceros bezoarticus (Linnaeus, 1758): Contribution to the knowledge of the historical distribution in Santa Catarina, southern Brazil. Check List. v.7, n.2, p.146-148, 2011.

TORTATO, F. R.; TESTONI, A. F.; ALTHOFF, S. L. Mastofauna terrestre da Reserva Biológica Estadual do Sassafrás, Doutor Pedrinho, Santa Catarina, Sul do Brasil. Biotemas, v.27, n.3, p.123-129, 2014.

VIBRANS, A. C.; MCROBERTS, R. E.; LINGNER, D. V. D.; NICOLETTI, A. L.; MOSER, P. Extensão original e remanescentes da Floresta Ombrófila Densa em Santa Catarina. In: VIBRANS, A.; SEVEGNANI, L.; GASPER, A. L. D.; LINGNER, D. V. D. Inventário Florístico Florestal de Santa Catarina, Vol IV, Floresta Ombrófila Densa. Blumenau: Edifurb, 2013, p.25-33.

WILSON, D. E.; REEDER, D. M. Mammals species of the World. A taxonomic and geographic reference. Baltimore: Johns Hopkins University Press, 2005, 2142p. 


\section{TABELAS}

Tabela 1. Lista de taxa de mamíferos de médio, grande porte e morcegos com ocorrência confirmada nos respectivos municípioscomponentes da região sul do estado de Santa Catarina, sul do Brasil, conforme numeração atribuída na Figura 1, onde: $\mathrm{X}=$ registros inéditos com base em amostragem de campo e registros oportunísticos; A = dados oriundos de Cheremet al. (2004); B = dados oriundos de Cherem (2005); C = Zocche et al. (2010); D = Carvalho et al. (2013); E = Carvalho et al. (2009); F = IPAT-UNESC (2006); G = Mattia (2016); F = Species Link. 01 - Praia Grande; 02 - São João do Sul; 03 - Passos de Torres; 04 - Jacinto Machado; 05 - Santa Rosa do Sul; 06 - Sombrio; 07 - Balneário Gaivota; 08 - Timbé do Sul; 09 - Turvo; 10 - Ermo; 11 - Araranguá; 12 - Balneário Arroio do Silva; 13 - Morro Grande; 14 - Meleiro; 15 - Maracajá; 16 - Nova Veneza; 17 - Forquilhinha; 18 - Içara; 19 - Siderópolis; 20 - Criciúma; 21 - Morro da Fumaça; 22 - Sangão; 23 Jaguaruna; 24 - Treviso; 25 - Urussanga; 26 - Cocal do Sul; 27 - Pedras Grandes; 28 - Treze de Maio; 29 - Tubarão; 30 - Laguna; 31 - Lauro Muller; 32 - Orleans; 33 - São Ludgero; 34 - Gravatal; 35 - Capivari de Baixo; 36 - Grão Pará; 37 - Braço do Norte; 38 - Armazém; 39 - Imaruí; 40 - Imbituba; 41 - Santa Rosa de Lima; 42 - Rio Fortuna; 43 - São Martinho; 44 - Garopaba.

\section{TAXA}

\section{MUNICÍPIOS DA REGIÃO SUL CATARINENSE}

\section{ARTIODACTYLA}

Mazama americana

Mazama nana

Ozotoceros bezoarticus

Pecari tajacu

Tayassu tajacu

CARNIVORA

Leopardus guttulus

Leopardus pardalis

Leopardus wiedii

Puma concolor

Puma yagouarondi

Cerdocyon thous

Lycalopex gymnocercus

Eira barbara

Lontra longicaudis

Nasua nasua

$\begin{array}{llllllllllll}1 & 2 & 3 & 4 & 5 & 6 & 7 & 8 & 9 & 10 & 11 & 14\end{array}$

Procyon cancrivorus

\begin{tabular}{|c|c|c|c|c|c|c|c|c|c|c|c|c|c|c|c|c|c|c|c|c|c|c|c|c|c|c|c|c|c|c|c|c|}
\hline - & - & - & - & - & - & - & - & - & - & - & A & - & - & - & - & - & - & - & - & - & - & - & - & - & - & - & - & - & - & - & - & - \\
\hline - & - & - & - & - & - & - & - & $X$ & - & - & - & - & $X$ & - & - & - & - & - & - & - & - & - & - & - & - & - & - & - & - & - & - & - \\
\hline- & - & - & - & - & - & - & - & - & - & - & - & - & - & - & - & - & - & - & - & - & - & - & - & - & - & $X$ & - & A & - & - & - & - \\
\hline- & - & - & - & - & - & - & A & - & - & - & A & - & A & - & - & $X$ & - & - & - & - & 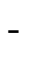 & - & - & - & - & - & $X$ & - & - & & - & - \\
\hline - & - & - & - & - & - & - & - & - & - & - & - & - & - & - & - & . & - & - & - & - & - & - & - & - & - & $X$ & - & - & - & & - & - \\
\hline - & - & - & - & - & - & - & $X$ & $X$ & - & $X$ & $X$ & $X$ & $X$ & $X$ & - & $X$ & $X$ & $X$ & $X$ & $X$ & - & $X$ & - & - & - & $X$ & $X$ & A & - & & - & - \\
\hline- & - & - & - & - & - & - & - & - & - & - & - & - & $X$ & - & - & $X$ & - & - & $X$ & - & 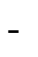 & - & - & - & - & - & $X$ & - & - & - & - & - \\
\hline- & - & - & - & - & - & - & - & $X$ & - & $X$ & $X$ & $X$ & $X$ & - & - & $X$ & $X$ & $X$ & $X$ & $\mathrm{G}$ & - & - & - & - & - & - & $X$ & A & - & - & - & - \\
\hline A & - & - & - & - & - & - & - & - & - & - & A & - & $X$ & - & - & $X$ & - & - & $X$ & - & - & - & - & - & - & - & $X$ & - & - & - & - & - \\
\hline- & - & - & - & - & - & - & - & - & - & - & - & - & $X$ & - & - & - & - & - & $X$ & - & - & - & - & - & - & - & - & - & - & - & - & - \\
\hline- & - & - & - & - & $X$ & - & $X$ & $X$ & $X$ & $X$ & $X$ & $X$ & $X$ & $X$ & - & $X$ & $\mathrm{G}$ & $X$ & $X$ & $\mathrm{G}$ & - & $X$ & $X$ & $X$ & X & $\mathrm{G}$ & $X$ & - & - & - & - & $\mathrm{A}$ \\
\hline- & - & - & - & - & - & - & - & - & - & - & - & - & - & - & - & - & - & - & - & - & - & - & - & - & - & $X$ & - & - & - & - & - & - \\
\hline A & - & - & - & - & - & - & - & - & - & $X$ & - & $X$ & - & - & - & $X$ & - & - & $X$ & $\mathrm{G}$ & - & - & - & - & - & $\mathrm{G}$ & $X$ & - & - & - & - & - \\
\hline- & $X$ & - & - & - & - & - & $X$ & - & - & A & - & - & $X$ & - & - & $X$ & - & $X$ & $X$ & $X$ & - & $X$ & - & - & - & - & $X$ & - & - & - & - & - \\
\hline- & - & - & - & - & - & - & - & - & - & - & - & - & - & - & - & $X$ & - & - & $X$ & $X$ & - & $X$ & - & - & - & $\mathrm{G}$ & $\mathrm{G}$ & - & - & - & - & - \\
\hline- & - & - & - & - & $X$ & - & $X$ & $X$ & - & $X$ & $X$ & $X$ & $X$ & - & $X$ & $X$ & $X$ & $X$ & $X$ & $\mathrm{G}$ & - & $X$ & - & - & A & $X$ & $X$ & - & $X$ & - & $X$ & - \\
\hline
\end{tabular}




\begin{tabular}{|c|c|c|c|c|c|c|c|c|c|c|c|c|c|c|c|c|c|c|c|c|c|c|c|c|c|c|c|c|c|c|c|c|c|}
\hline \multirow{2}{*}{ TAXA } & \multicolumn{33}{|c|}{ MUNICÍPIOS DA REGIÃO SUL CATARINENSE } \\
\hline & 1 & 2 & 3 & 4 & 5 & 6 & 7 & 8 & 9 & 10 & 11 & 14 & 15 & 16 & 17 & 18 & 19 & 20 & 23 & 24 & 25 & 26 & 27 & 28 & 29 & 30 & 31 & 32 & 33 & 36 & 42 & 43 & 44 \\
\hline Conepatus chinga & - & - & - & - & - & - & - & $X$ & - & - & - & - & - & - & - & - & - & - & - & - & - & - & - & - & - & - & $\mathrm{X}$ & - & - & - & - & - & - \\
\hline \multicolumn{34}{|l|}{ CHIROPTERA } \\
\hline Anoura caudifer & - & - & - & - & A & - & - & $X$ & - & - & - & - & - & A & - & - & A & - & - & $\mathrm{F}$ & $X$ & - & $\mathrm{D}$ & - & - & - & - & - & - & - & - & - & - \\
\hline Anoura geoffroyi & - & - & - & - & - & - & - & $X$ & - & - & - & - & - & - & - & - & - & - & - & $\mathrm{F}$ & $X$ & - & $\mathrm{D}$ & - & - & - & - & - & - & - & - & - & - \\
\hline Artibeus fimbriatus & - & - & - & A & - & - & - & $X$ & - & - & - & - & - & $X$ & - & - & - & $X$ & $\mathrm{E}$ & - & $X$ & - & $\mathrm{D}$ & A & - & - & - & - & - & - & - & - & - \\
\hline Artibeus lituratus & - & - & - & A & A & - & - & $\mathrm{X}$ & $\mathrm{X}$ & - & - & - & - & $X$ & - & - & $X$ & $X$ & $\mathrm{E}$ & $\mathrm{X}$ & $X$ & - & $\mathrm{D}$ & $\mathrm{A}$ & - & - & - & - & - & - & - & - & - \\
\hline Artibeus obscurus & - & - & - & - & - & - & - & $\mathrm{X}$ & $\mathrm{X}$ & - & - & - & - & - & - & - & $\mathrm{X}$ & - & $\mathrm{E}$ & - & $X$ & - & $\mathrm{D}$ & - & - & - & - & - & - & - & - & - & - \\
\hline Carollia perspicillata & - & - & - & - & - & - & - & $\mathrm{X}$ & $X$ & - & - & - & - & A & - & - & $X$ & - & - & $\mathrm{X}$ & $\mathrm{X}$ & - & $\mathrm{D}$ & - & - & - & - & - & - & - & - & - & - \\
\hline Chrotopterus auritus & - & - & - & - & - & - & - & - & - & - & - & - & - & - & - & - & - & - & - & - & - & - & $\mathrm{D}$ & - & - & - & - & - & - & - & - & - & - \\
\hline Desmodus rotundus & - & - & - & - & - & - & - & $\mathrm{X}$ & - & - & - & - & - & - & - & - & - & - & $\mathrm{E}$ & $\mathrm{F}$ & $X$ & - & $\mathrm{D}$ & - & - & - & - & $X$ & - & - & - & - & - \\
\hline Diphylla ecaudata & - & - & - & - & - & - & - & - & - & - & - & - & - & - & - & - & - & - & - & - & - & - & $\mathrm{D}$ & - & - & - & - & - & - & - & - & - & - \\
\hline Glossopaga soricina & - & - & - & - & - & - & - & $X$ & - & - & - & - & - & - & - & - & - & $\mathrm{X}$ & $\mathrm{X}$ & - & - & - & $\mathrm{D}$ & - & - & - & - & - & - & - & - & - & - \\
\hline Mimon bennettii & - & - & - & - & - & - & - & - & - & - & - & - & - & - & - & - & - & - & - & - & - & - & $\mathrm{D}$ & - & - & - & - & - & - & - & - & - & - \\
\hline Platyrrhinus recifinus & - & - & - & - & - & - & - & - & - & - & - & - & - & - & - & - & - & - & - & - & - & - & $\mathrm{D}$ & - & - & - & - & - & - & - & - & - & - \\
\hline Pygoderma bilabiatum & - & - & - & - & - & - & - & - & - & - & - & - & - & - & - & - & - & - & - & $\mathrm{F}$ & - & - & $\mathrm{D}$ & - & - & - & - & - & - & - & - & - & - \\
\hline Sturnira lilium & - & - & - & - & A & - & - & $\mathrm{X}$ & $\mathrm{X}$ & - & - & - & - & $X$ & - & - & $X$ & $X$ & $\mathrm{E}$ & $\mathrm{X}$ & $X$ & - & $\mathrm{D}$ & - & - & - & - & - & - & - & - & - & - \\
\hline Sturnira tildae & - & - & - & - & - & - & - & - & - & - & - & - & - & - & - & - & - & - & $X$ & - & - & - & $\mathrm{D}$ & - & - & - & - & - & - & - & - & - & - \\
\hline Vampyressa pusilla & - & - & - & - & - & - & - & $\mathrm{X}$ & - & - & - & - & - & $\mathrm{A}$ & - & - & - & - & - & - & - & - & $\mathrm{D}$ & - & - & - & - & - & - & - & - & - & - \\
\hline Molossus molossus & - & - & - & - & - & - & - & $\mathrm{C}$ & - & - & - & - & - & $\mathrm{A}$ & $\mathrm{G}$ & - & $\mathrm{C}$ & $X$ & - & - & - & - & $X$ & - & - & - & - & - & - & - & - & - & - \\
\hline Tadarida brasiliensis & - & - & - & - & - & - & - & $\mathrm{C}$ & - & - & - & - & - & - & - & - & $\mathrm{C}$ & $X$ & - & - & $X$ & - & - & - & A & - & - & - & - & - & - & - & - \\
\hline Noctilio leporinus & - & - & - & - & - & - & - & - & - & - & - & - & - & - & - & - & - & - & - & - & $X$ & - & $X$ & - & - & - & - & - & - & - & - & - & - \\
\hline Eptesicus brasiliensis & - & - & - & - & - & - & - & $\mathrm{X}$ & - & - & - & - & - & - & - & - & - & $\mathrm{X}$ & - & - & - & - & $\mathrm{D}$ & - & - & - & - & - & - & - & - & - & - \\
\hline Eptesicus diminutus & - & - & - & - & - & - & - & $X$ & $\mathrm{X}$ & - & - & - & - & - & $\mathrm{G}$ & - & $\mathrm{C}$ & $X$ & $\mathrm{E}$ & $X$ & - & - & $\mathrm{D}$ & - & - & - & - & - & - & - & - & - & - \\
\hline Eptesicus furinalis & - & - & - & - & - & - & - & $\mathrm{X}$ & $\mathrm{X}$ & - & - & - & - & - & - & - & - & - & $X$ & - & - & - & $\mathrm{D}$ & - & - & - & - & - & - & - & - & - & - \\
\hline Lasiurus blossevillii & - & - & - & - & - & - & - & $X$ & - & - & - & - & - & - & - & - & - & $X$ & $\mathrm{E}$ & - & - & - & $\mathrm{D}$ & - & - & - & - & - & - & - & - & - & - \\
\hline Lasiurus cinereus & - & - & - & - & - & - & - & - & - & - & - & - & - & - & - & - & - & - & - & - & - & - & - & - & $\mathrm{X}$ & - & - & - & - & - & - & - & - \\
\hline Lasiurus ega & - & - & - & - & - & - & - & - & - & - & - & - & - & - & - & - & - & - & $\mathrm{X}$ & - & $\mathrm{G}$ & - & - & - & - & - & - & - & - & - & - & - & - \\
\hline Myotis dinellii & - & - & - & - & - & - & - & - & - & - & - & - & - & - & - & - & - & - & - & - & - & - & $\mathrm{D}$ & - & - & - & - & - & - & - & - & - & - \\
\hline
\end{tabular}




\begin{tabular}{|c|c|c|c|c|c|c|c|c|c|c|c|c|c|c|c|c|c|c|c|c|c|c|c|c|c|c|c|c|c|c|c|c|c|}
\hline \multirow{2}{*}{ TAXA } & \multicolumn{33}{|c|}{ MUNICÍPIOS DA REGIÃO SUL CATARINENSE } \\
\hline & 1 & 2 & 3 & 4 & 5 & 6 & 7 & 8 & 9 & 10 & 11 & 14 & 15 & 16 & 17 & 18 & 19 & 20 & 23 & 24 & 25 & 26 & 27 & 28 & 29 & 30 & 31 & 32 & 33 & 36 & 42 & 43 & 44 \\
\hline Myotis levis & - & - & - & - & - & - & - & $X$ & - & - & - & - & - & $\mathrm{A}$ & - & - & - & - & $\mathrm{X}$ & - & $\mathrm{X}$ & - & - & - & - & - & - & - & - & - & - & - & - \\
\hline Myotis nigricans & - & - & - & - & - & - & - & $X$ & - & - & - & - & - & $X$ & - & - & $X$ & $X$ & $\mathrm{E}$ & $X$ & $X$ & - & $\mathrm{D}$ & - & - & - & - & - & - & - & - & - & - \\
\hline Myotis riparius & - & - & - & - & A & - & - & $\mathrm{X}$ & $\mathrm{X}$ & - & - & - & - & A & - & - & - & $X$ & $\mathrm{E}$ & - & - & - & $\mathrm{D}$ & - & - & - & - & - & - & - & - & - & - \\
\hline Myotis ruber & - & - & - & - & - & - & - & $X$ & - & - & - & - & - & - & - & - & $X$ & $X$ & - & $\mathrm{F}$ & G & - & $\mathrm{D}$ & - & - & - & - & - & - & - & - & - & - \\
\hline \multicolumn{34}{|l|}{ CINGULATA } \\
\hline Cabassous tatouay & - & - & - & - & - & - & - & - & - & - & - & - & - & - & - & - & - & G & $\mathrm{X}$ & - & $\mathrm{X}$ & - & - & - & - & - & - & G & - & - & - & - & - \\
\hline Euphractus sexcinctus & - & - & - & - & - & - & - & - & - & - & - & - & - & - & - & - & - & - & - & - & - & - & - & - & - & - & - & $\mathrm{X}$ & - & - & - & - & - \\
\hline Dasypus novemcinctus & - & - & - & - & - & - & - & - & - & - & $X$ & $X$ & - & $X$ & - & - & $X$ & G & $X$ & $\mathrm{X}$ & $\mathrm{G}$ & $\mathrm{G}$ & $X$ & - & - & $X$ & $\mathrm{G}$ & G & - & $X$ & - & - & - \\
\hline Dasypus hybridus & - & - & - & - & - & - & - & - & - & - & - & - & - & - & - & - & - & - & - & - & $\mathrm{X}$ & $X$ & - & - & - & - & - & - & - & - & - & - & - \\
\hline \multicolumn{34}{|l|}{ DIDELPHIMORPHIA } \\
\hline Didelphis albiventris & - & - & - & - & - & A & - & $\mathrm{X}$ & B & - & $\mathrm{X}$ & $\mathrm{X}$ & A & $X$ & - & $X$ & $\mathrm{X}$ & G & $\mathrm{X}$ & $\mathrm{F}$ & $\mathrm{G}$ & $\mathrm{G}$ & $X$ & - & - & $X$ & $\mathrm{G}$ & G & - & - & - & - & - \\
\hline Didelphis aurita & - & - & - & - & - & - & - & - & - & - & - & - & - & - & - & - & B & $X$ & - & $X$ & $\mathrm{G}$ & - & $X$ & - & - & A & $\mathrm{G}$ & G & A & - & - & $X$ & - \\
\hline \multicolumn{34}{|l|}{ LAGOMORPHA } \\
\hline Lepus europaeus & - & - & - & - & - & - & - & $\mathrm{X}$ & - & - & - & - & - & $X$ & - & - & - & $X$ & $\mathrm{X}$ & - & $\mathrm{G}$ & - & $X$ & - & - & - & $X$ & $\mathrm{X}$ & - & - & - & - & - \\
\hline $\begin{array}{l}\text { Sylvilagus brasiliensis } \\
\text { PILOSA }\end{array}$ & - & - & - & - & - & A & - & - & - & - & - & - & - & - & - & - & - & - & - & - & - & - & - & - & - & - & - & - & - & - & - & - & - \\
\hline $\begin{array}{l}\text { Tamandua tetradactyla } \\
\text { PRIMATES }\end{array}$ & A & - & - & - & - & A & - & - & - & - & - & - & $X$ & $X$ & - & - & $X$ & A & - & $X$ & $X$ & - & - & - & - & - & $X$ & $X$ & A & - & - & - & - \\
\hline Alouatta guariba clamitans & - & - & - & A & - & - & - & - & - & - & - & - & - & $X$ & - & - & $X$ & $\mathrm{X}$ & - & $X$ & $X$ & - & $\mathrm{X}$ & - & - & - & - & $\mathrm{X}$ & - & - & - & $\mathrm{X}$ & - \\
\hline Sapajus apella & - & - & $X$ & - & - & - & - & - & - & - & - & - & $X$ & $X$ & - & - & $X$ & $\mathrm{X}$ & - & $\mathrm{X}$ & - & - & - & - & - & - & - & - & - & - & - & - & - \\
\hline \multicolumn{34}{|l|}{ RODENTIA } \\
\hline Cuniculus paca & - & - & - & - & - & - & - & $\mathrm{X}$ & - & - & - & - & - & - & - & - & $X$ & - & - & $X$ & - & - & $X$ & - & - & - & - & - & A & - & - & A & A \\
\hline Dasyprocta azarae & - & - & - & - & - & - & - & - & - & - & - & - & - & $X$ & - & $\mathrm{X}$ & $X$ & - & $\mathrm{X}$ & $\mathrm{X}$ & - & - & $X$ & - & - & - & - & - & - & - & - & - & A \\
\hline Sphiggurus villosus & - & - & - & - & - & - & - & $X$ & $\mathrm{X}$ & - & $\mathrm{X}$ & $X$ & - & $X$ & - & $X$ & $\mathrm{X}$ & $\mathrm{G}$ & - & $\mathrm{X}$ & $\mathrm{G}$ & $\mathrm{G}$ & $X$ & - & - & - & - & $\mathrm{G}$ & - & - & - & - & - \\
\hline Hydrochoerus hydrochaeris & - & - & $X$ & - & - & $X$ & $\mathrm{X}$ & - & - & - & $X$ & A & $X$ & $X$ & - & $X$ & $X$ & $\mathrm{X}$ & $X$ & $\mathrm{~F}$ & $\mathrm{G}$ & $X$ & $X$ & - & $X$ & $\mathrm{X}$ & - & $X$ & A & - & - & $X$ & - \\
\hline Myocastor coypus & - & - & - & - & - & $\mathrm{X}$ & - & - & $X$ & - & $\mathrm{X}$ & - & $\mathrm{X}$ & $\mathrm{X}$ & - & $X$ & $\mathrm{~B}$ & $\mathrm{X}$ & A & - & $\mathrm{G}$ & - & - & - & - & $X$ & - & $\mathrm{G}$ & - & - & - & - & - \\
\hline Riqueza por município & 3 & 1 & 2 & 3 & 4 & 7 & 1 & 30 & 15 & 1 & 11 & 11 & 10 & 30 & 5 & 6 & 31 & 27 & 25 & 30 & 32 & 5 & 40 & 3 & 4 & 7 & 14 & 22 & 7 & 2 & 3 & 5 & 3 \\
\hline
\end{tabular}


Tabela 2. Relação de taxa da fauna de mamíferos terrestre de médio, grande porte e morcegos, listados em alguma das categorias de ameaça para âmbito global, nacional e/ou estadual registrados na região sul de Santa Catarina, onde: $\mathrm{DD}=$ dados deficientes; $\mathrm{NT}=$ quase ameaçada; $\mathrm{CR}=$ criticamente em perigo; $\mathrm{VU}=$ vulnerável; $\mathrm{EN}=$ em perigo; IUCN $=$, International Unionfor Conservation of Nature; MMA $=$ Ministério do Meio Ambiente; CONSEMA = Conselho Estadual do Meio Ambiente/SC.

TAXA

CATEGORIAS DE AMEAÇAS/ENTIDADES

ARTIODACTYLA

Mazama americana

Mazama nana

Ozotoceros bezoarticus

Pecari tajacu

Tayassu tajacu

CHIROPTERA

Diphylla ecaudata

Myotis ruber

Sturnira tildae

FELIDAE

Leopardus guttulus

Leopardus pardalis

Leopardus wiedii

Puma concolor

Puma yagouarondi

PRIMATES

Alouatta guariba clamitans

RODENTIA

Cuniculus paca

IUCN

MMA CONSEMA/SC

Total de espécies

\begin{tabular}{ccc} 
DD & - & EN \\
DD & VU & VU \\
NT & VU & VU \\
- & - & VU \\
- & VU & CR \\
& & \\
- & - & EN \\
NT & - & \\
- & - & VU \\
& & \\
- & VU & - \\
- & - & EN \\
NT & VU & - \\
- & VU & VU \\
- & VU & - \\
& & \\
- & VU & VU \\
& - & - \\
- & - & VU \\
\hline $\mathbf{5}$ & $\mathbf{8}$ & $\mathbf{1 1}$ \\
\hline & &
\end{tabular}

